\title{
Randomised comparison of the effects of nicardipine and esmolol on coronary artery wall stress: implications for the risk of plaque rupture
}

\author{
M J A Williams, C J S Low, G T Wilkins, R A H Stewart
}

\begin{abstract}
Objective-To determine whether the $\beta$ blocker esmolol reduces coronary artery wall stress more than the short acting dihydropyridine calcium antagonist nicardipine.

Design-Randomised double blind placebo controlled trial.

Setting-Tertiary cardiology centre.

Patients-Patients with coronary artery disease.

Interventions - 20 patients were randomised double blind to an infusion of nicardipine $(\mathrm{n}=10)$ or esmolol $(\mathrm{n}=10)$ titrated to reduce systolic blood pressure by $20 \mathrm{~mm} \mathrm{Hg}$.

Main outcome measures-Peak systolic wall circumferential stress.

Results-Esmolol reduced peak coronary stress by a mean of $0.17 \times 10^{6} \mathrm{dyn} / \mathrm{cm}^{2}(95 \%$ confidence interval (CI) 0.14 to $0.21 \times 10^{6} \mathrm{dyn} / \mathrm{cm}^{2}$ ) compared with a reduction of $0.07 \times 10^{6} \mathrm{dyn} / \mathrm{cm}^{2}\left(95 \%\right.$ CI 0.05 to $\left.0.10 \times 10^{6} \mathrm{dyn} / \mathrm{cm}^{2}\right)$ after nicardipine. Peak systolic radius was reduced by $0.04 \mathrm{~mm}$ ( $95 \%$ CI 0.03 to $0.06 \mathrm{~mm}$ ) after esmolol compared with an increase of $0.08 \mathrm{~mm}(95 \%$ CI 0.05 to $0.10 \mathrm{~mm})$ after nicardipine. Heart rate increased by $11.5 \mathrm{beats} / \mathrm{min}$ (95\% CI 6.9 to 16.2 beats/min) after nicardipine and decreased by 5.3 beats $/ \mathrm{min}$ (95\% CI 1.9 to 8.6 beats/min) after esmolol.
\end{abstract}

Conclusions-Intravenous esmolol is more effective than nicardipine at reducing circumferential coronary artery wall stress.

(Heart 2000;84:377-382)

Keywords: $\beta$ blockers; calcium channel antagonists; mechanics; coronary disease

It is established that $\beta$ blockers reduce mortality following myocardial infarction. ${ }^{1}$ In contrast there is controversial evidence that short acting dihydropyridine calcium antagonists do not decrease $\mathrm{e}^{3}$ and may even increase the risk of cardiovascular events..$^{4-6}$ The pathophysiological mechanisms which might account for different rates of ischaemic coronary events with these two drug classes are uncertain. Proposed mechanisms for the possible adverse effects of calcium antagonists include sympathetic activation, proischaemia, negative inotropy, and reflex tachycardia. ${ }^{4} 78$

Plaque rupture is the most common initial event in unstable angina and myocardial infarction. ${ }^{9}$ The risk of plaque rupture is determined by the intrinsic properties of the plaque and extrinsic forces acting on it. ${ }^{10}$ It is possible that $\beta$ blockers and dihydropyridine calcium antagonists, while both lowering blood pressure, have different effects on coronary artery wall stress, a possible determinant of plaque rupture. ${ }^{11}$ In this study we test the hypothesis that, for an equivalent reduction in systolic blood pressure, the $\beta$ blocker esmolol reduces coronary artery wall stress more than the short acting dihydropyridine calcium antagonist nicardipine.

\section{Methods}

STUDY PATIENTS

Eligible patients had single vessel coronary artery disease, were scheduled to have elective coronary angioplasty, and had minimal disease of the proximal left anterior descending or left circumflex coronary artery. Patients were excluded if there were contraindications to receiving nicardipine or esmolol, including asthma, severe valvar disease, congestive heart failure, and impaired hepatic or renal function. Vasoactive drugs were withheld for at least 24 hours before the investigation. Patients were randomised after a successful baseline intravascular ultrasound study had been completed. Written informed consent was obtained from all participating patients and the study was approved by the regional health authority ethics committee.

\section{CATHETERISATION PROCEDURE}

After administration of $10000 \mathrm{U}$ heparin, coronary angiography was performed through the right femoral artery using 6 French diagnostic catheters. Nitrates or other non-study vasoactive drugs were not given before or during the research protocol. An 8 French left Judkins type guiding catheter was then used for insertion of either a 3 French or a 3.5 French, $30 \mathrm{MHz}$ intravascular ultrasound (IVUS) imaging catheter (Boston Scientific, Watertown, Massachusetts, USA) into the proximal left anterior descending or proximal circumflex coronary artery. Arterial pressure was measured with a 2 French micromanometer (Millar Mikro-Tip SPC-320, Millar Instruments, Houston, Texas, USA) inserted from the left femoral artery through a 6 French left diagnostic Judkins type catheter, with its tip located adjacent to the left main coronary artery ostium. 

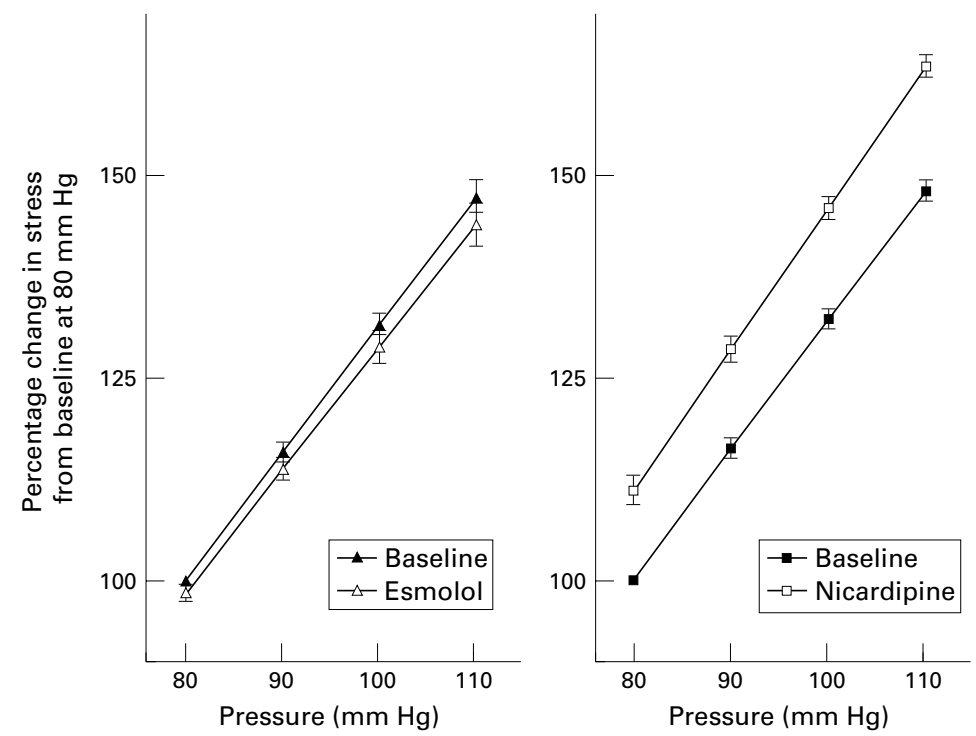

Figure 1 Relation between circumferential wall stress and arterial pressure before and after esmolol (left) and nicardipine (right). Results are presented as means with SEM. Circumferential wall stress is expressed as the percent change from baseline stress estimated at $80 \mathrm{~mm} \mathrm{Hg}$. Esmolol reduced coronary artery stress $(p=0.024)$ while nicardipine increased it $(p=0.001)$. two minutes, and a repeat ultrasound study was performed in the same position as the baseline study.

\section{DATA ANALYSIS}

The intravascular ultrasound images were analysed off-line to determine coronary artery luminal area and wall thickness by a single observer blinded to treatment allocation. Video frames were digitised using the built in digitise of a PowerMac 8500 computer and measured using NIH Image 1.60, a public domain program from the US National Institutes of Health. Peak systolic, late systolic, early diastolic, mid-diastolic, and late diastolic images and their simultaneously acquired pressures were measured at matching time intervals for three sequential cardiac cycles. The luminal area was determined by tracing the lumen-intima interface for each of these phases of the cardiac cycle and the results recorded as the mean of three cardiac cycles. Wall thickness was measured as the distance from the lumenintima interface to the media-adventitia interface. ${ }^{12}$ This measurement does not include the thickness of the arterial adventitia, which represents a small proportion of the arterial wall thickness in an atherosclerotic coronary artery. ${ }^{13}$ Five measurements of wall thickness were made at peak systole and averaged for three cardiac cycles. Wall thickness for other phases of the cardiac cycle was calculated by assuming the vessel wall was incompressible and that wall volume remained constant through the cardiac cycle. ${ }^{14}{ }^{15}$ Heart rate was calculated from the ECG after the measurement of all ultrasound images to maintain blinding of treatment allocation.

\section{CALCULATIONS}

Measurements of arterial area (A) were used to derive the inner arterial radius $(r)$ :

$\mathrm{r}=\sqrt{\frac{\mathrm{A}}{\pi}}$

artery pressure waveform and coincident arterial images were acquired using a HewlettPackard Sonos 100 IVUS machine and recorded on Super-VHS videotape for subsequent analysis. The ECG and coronary pressures were recorded as a digital file using a MacLab 8/s hardware unit (AD Instruments, Castle Hill, NSW, Australia).

STUDY PROTOCOL

Patients were randomised blockwise to treatment allocation using a random number list. The allocation schedule was kept in a numbered series of sealed opaque envelopes. All patients randomised completed the study protocol. Drug infusions were prepared by staff not involved with the trial and were given double blind. After the baseline intravascular ultrasound study, patients received an infusion of either esmolol $(500 \mu \mathrm{g} / \mathrm{min})$ or nicardipine $(500 \mu \mathrm{g} / \mathrm{min})$ to reduce the systolic blood pressure by approximately $20 \mathrm{~mm} \mathrm{Hg}$. After achieving the target blood pressure reduction (nicardipine, 5.8 (2.0) minutes; esmolol, 11.5 (2.9) minutes (mean (SD)) the drug infusion was continued to maintain a steady state for
Circumferential wall stress $(\sigma)$ was calculated by the following formula:

$\sigma=\frac{\mathrm{P} \times \mathrm{r}}{\mathrm{h}}$

where $\mathrm{P}$ is arterial pressure, $\mathrm{r}$ is the arterial radius, and $h$ is arterial wall thickness.

\section{Pressure-stress relation}

To assess the effects of drug treatment on corblood pressure, a pressure-stress relation was calculated before and after drug treatment. The method of calculating the pressure-stress relation has been reported. ${ }^{13}$ A mathematical model was used to fit arterial pressure and lumen area data, and this model was used to derive pressure-stress curves which would permit comparison of the effects of study drugs on coronary stress over a common range of pressures. This allows direct comparison of the effects of each drug on coronary stress, onary stress while controlling for the effects of 
Table 1 Baseline characteristics of the patients

\begin{tabular}{|c|c|c|}
\hline & $\begin{array}{l}\text { Nicardipine } \\
(n=10)\end{array}$ & $\begin{array}{l}\text { Esmolol } \\
(n=10)\end{array}$ \\
\hline \multicolumn{3}{|l|}{ Demography } \\
\hline Age (years) (mean (SD)) & $54.3(8.8)$ & $59.0(5.9)$ \\
\hline Male/female & $8 / 2$ & $6 / 4$ \\
\hline \multicolumn{3}{|l|}{ Risk factors } \\
\hline Hypertension & 4 & 2 \\
\hline Hypercholesterolaemia & 8 & 4 \\
\hline Diabetes & 3 & 1 \\
\hline \multicolumn{3}{|l|}{ Previous drug treatment } \\
\hline$\beta$ Blocker & 8 & 7 \\
\hline Calcium antagonist & 6 & 7 \\
\hline
\end{tabular}

mediated by their effect on arterial radius independent of passive changes in arterial radius related to changes in blood pressure.

Arterial pressure and area data points were plotted and fitted with least squares regression using the arctangent function described by Langewouters and colleagues. ${ }^{16}$

$\mathrm{A}=\alpha\left[\frac{1}{2}+\frac{1}{\pi} \tan ^{-1}\left(\frac{\mathrm{P}-\boldsymbol{\beta}}{\gamma}\right)\right]$

where $\mathrm{A}$ is arterial area, $\mathrm{P}$ is arterial pressure, and $\alpha, \beta$, and $\gamma$ are three optimal fit parameters.

All individual pressure-area data fit this arc tangent model with $R^{2}>0.99$. The individual pressure-area functions were then used to calculate pressure-stress curves using equations (1) and (2) for each patient before and after drug treatment. Points on the fitted curves for individual subjects were averaged every $10 \mathrm{~mm} \mathrm{Hg}$ in the range from $80 \mathrm{~mm} \mathrm{Hg}$ to $110 \mathrm{~mm} \mathrm{Hg}$ (this range of pressures included values recorded from all patients) and the group mean values plotted before and after drug treatment (fig 1).

END POINTS

The primary study end point was change in peak circumferential coronary wall stress. The secondary end point was the change in circumferential stress controlled for pressure.

STATISTICS

The sample size calculation was based on the expectation that differences in coronary stress would be related to changes in vessel radius, assuming similar wall thickness and blood pressure. Intracoronary nicardipine has been reported to increase vessel area by $11 \%,{ }^{17}$ which represents an increase in vessel radius of approximately $5 \%$. For the primary end point of coronary circumferential stress the study (two groups of 10 patients) had a 95\% power of detecting a difference in circumferential stress of 5\% (SD 3\%), assuming a two sided type I error level of 0.05 . The statistical analysis was performed with SPSS. The results for the two treatment groups were compared with unpaired Student's $t$ test for normally distributed variables and the Wilcoxon rank sum test for other variables. Fisher's exact test was used for categorical variables. The pressure-stress curves generated at baseline and after drug administration were compared with repeated measures analysis of variance. Measurement variability was assessed using the method of Bland and Altman ${ }^{18}$ and was expressed as the mean (SD) of the differences in measurements. The effect of treatment on outcome measures is presented as mean change from baseline and 95\% confidence interval (CI).

\section{Results}

There were 39 patients screened for participation in the study. Of these, 16 had unsuitable coronary anatomy and three did not have a technically satisfactory baseline intravascular ultrasound study. Twenty patients were randomised to either nicardipine $(n=10)$ or esmolol $(n=10)$. The baseline clinical characteristics (table 1) and haemodynamic measurements (table 2) were similar for each treatment group. Intravascular ultrasound studies were performed in the proximal left anterior descending artery in 14 patients (nicardipine 7, esmolol 7) and the proximal left circumflex artery in six patients (nicardipine 3, esmolol 3). Intravascular measurements of systolic arterial area, wall thickness, circumferential stress, and coronary stress index were similar for the two treatment groups at baseline (table 2).

Changes in heart rate and arterial indices are presented as the mean change from baseline in

Table 2 Baseline haemodynamics and arterial measurements

\begin{tabular}{llll}
\hline & Nicardipine $(n=10)$ & Esmolol $(n=10)$ & $p$ Value \\
\hline Haemodynamics & $136.5(21.8)$ & $141.4(22.3)$ & 0.62 \\
Systolic blood pressure (mm Hg) & $83.6(16.0)$ & $78.3(9.8)$ & 0.39 \\
Diastolic blood pressure (mm Hg) & $70.0(17.1)$ & $70.9(9.2)$ & 0.89 \\
Heart rate (beats/min) & $0.38(0.14)$ & $0.37(0.11)$ & 0.91 \\
Arterial measurements & $7.86(2.44)$ & $8.67(2.68)$ & 0.54 \\
Arterial wall thickness (mm) & $1.56(0.26)$ & $1.64(0.26)$ & 0.49 \\
Peak systolic arterial area $\left(\mathrm{mm}^{2}\right)$ & $0.95(0.34)$ & $1.00(0.42)$ & 0.82 \\
Peak systolic arterial radius $\left(\mathrm{mm}^{2}\right)$ & & & \\
Peak wall stress $\left(10^{6} \mathrm{dyn} / \mathrm{cm}^{2}\right)$ & & & \\
\hline
\end{tabular}

Values are mean (SD).

Table 3 Change in heart rate and peak systolic arterial indices with a $20 \mathrm{~mm} \mathrm{Hg}$ decrease in systolic pressure after nicardipine or esmolol

\begin{tabular}{lllll}
\hline & \multicolumn{2}{l}{ Mean change from baseline $(95 \%$ CI) } & Difference $(95 \%$ CI) & $p$ Value \\
\cline { 2 - 3 } & Nicardipine $(n=10)$ & Esmolol $(n=10)$ & $16.8(11.5$ to 22.2$)$ & 0.0002 \\
Heart rate (beats $/ \mathrm{min})$ & $11.5(6.9$ to 16.2$)$ & $-5.3(-1.9$ to -8.6$)$ & $0.12(0.09$ to 0.15$)$ & 0.0002 \\
Systolic radius $(\mathrm{mm})$ & $0.08(0.05$ to 0.10$)$ & $-0.04(-0.03$ to -0.06$)$ & $0.10(0.06$ to 0.14$)$ & 0.0005 \\
Peak stress $\left(10^{6} \mathrm{dyn} / \mathrm{cm}^{2}\right)$ & $-0.07(-0.05$ to -0.10$)$ & $-0.17(-0.14$ to -0.21$)$ & 0.17 \\
\hline
\end{tabular}

Change from baseline $=$ post-drug value - baseline value . 
table 3. Analysis of changes in stress independent of blood pressure showed that esmolol minimally decreased coronary artery stress by $2-3 \%$ (fig 1). Most of the $18 \%$ reduction in peak wall stress after esmolol was caused by the $20 \mathrm{~mm} \mathrm{Hg}$ decrease in blood pressure. In contrast, after controlling for blood pressure nicardipine increased coronary stress by approximately $10 \%$ (fig 1 ) by causing vasodilatation. This increase offset the reduction in stress related to the $20 \mathrm{~mm} \mathrm{Hg}$ fall in blood pressure, and the net reduction in peak stress per cardiac cycle was $8 \%$ with nicardipine (table 3 ).

Measurement variability was assessed by measuring 30 randomly selected intravascular ultrasound images for vessel area and 15 images for wall thickness. The mean (SD) difference between two blinded measurements of coronary artery area performed by one observer was $0.02(0.15) \mathrm{mm}^{2}$. The intraobserver difference for measurements of wall thickness was $0.0013(0.0052) \mathrm{mm}$.

\section{Discussion}

In this study a $\beta$ blocker and a short acting dihydropyridine calcium antagonist both lowered blood pressure but had different effects on coronary artery circumferential wall stress. Necropsy studies have established that plaque rupture occurs in regions of the arterial wall with high levels of circumferential stress. ${ }^{11}$ It is likely that treatment which reduces coronary wall stress will reduce the long term risk of myocardial infarction and sudden death from coronary artery disease. This possibility is consistent with the clearly established benefits of $\beta$ blockade in reducing reinfarction and death in patients with coronary artery disease. ${ }^{1}$ A lesser reduction in coronary wall stress might conceivably contribute to the less favourable effects on cardiovascular events in clinical trials $^{4-619}$ and case-control studies ${ }^{20}$ of dihydropyridine calcium antagonists.

Previous investigators have suggested that sympathetic activation associated with reflex tachycardia and raised noradrenaline (norepinephrine) may explain the possible adverse effects of calcium antagonists. ${ }^{22}$ However, the precise mechanisms by which these factors increase the risk of cardiovascular events are uncertain. It is possible that sympathetic activation and increased heart rate increase cumulative coronary circumferential stress and therefore the risk of plaque rupture. Studies of materials fatigue show that a stress applied repetitively increases the likelihood of fracture. ${ }^{23}$ However, the precise relation between applied stress and cycle number required for plaque fracture in vivo is unknown. A role for heart rate in the risk of plaque rupture is supported by the good correlation between reduction in heart rate and decrease in mortality in patients with coronary heart disease treated with $\beta$ blockers. ${ }^{24}$ In addition, and in contrast to the dihydropyridine calcium antagonists, the non-dihydropyridine calcium antagonists verapamil and diltiazem, which slow heart rate, appear to reduce cardiovascular event rates. ${ }^{25}$
The coronary vasodilator effects of nicardipine acted to increase coronary stress by increasing vessel radius. However, peak coronary wall stress was reduced by nicardipine because the effect of reduction in systolic blood pressure was greater than the direct effect of coronary vasodilatation. This observation suggests that calcium antagonists which do not cause tachycardia may have a favourable effect on coronary artery wall stress. By comparison, $\beta$ blockers-which do not cause coronary vasodilatation-may be more effective at reducing coronary artery wall stress. In this study homogeneous arterial segments were studied, but both $\beta$ blockers ${ }^{26}$ and dihydropyridine calcium antagonists ${ }^{17}$ are known to have similar vasomotor effects in normal and diseased segments of artery.

Changes in coronary wall stress were measured at rest rather than during exercise, when myocardial infarction is more likely to be triggered. ${ }^{27}{ }^{28}$ In addition, the present study assessed acute rather than chronic effects of drug treatment. Reflex tachycardia with dihydropyridines decreases with chronic treatment and is less with long acting dihyropyridine calcium antagonists. The increase in heart rate approximates $3-4 \%$ during chronic treatment ${ }^{22}$ compared with a decrease in heart rate averaging $14 \%$ in 11 randomised trials of long term $\beta$ blockade. ${ }^{24}$ Significant differences in heart rate are therefore likely to persist with long term treatment and during the stress of exercise. However, the effects of long term treatment on coronary wall stress have not been studied and the relation between the acute changes in coronary wall stress measured in this study and the long term risk of plaque rupture is uncertain.

Estimation of mean coronary artery circumferential stress requires simultaneous measurements of arterial area and pressure. These measurements may be influenced by differences between aortic root and proximal artery pressure, vasoconstriction after introduction of the intravascular ultrasound catheter, and axial movement of the imaging catheter. ${ }^{29}$ The impact of each of these factors on the measurement of coronary artery wall stress is uncertain. However, the study design - which includes paired measurements before and after drug infusion and double blind random allocation of treatment - makes it unlikely that these factors would bias estimation of the treatment effect.

Several factors not assessed in the current study are likely to influence the risk of plaque rupture, including vessel wall structure, fibrous cap thickness, ${ }^{11} 3031$ lipid content, ${ }^{32}$ and local inflammation..$^{33}$ Finite element analysis, which assigns mechanical properties to individual elements of arterial wall and plaque based on micromechanical testing, has been used to calculate maximum circumferential stress in vitro. ${ }^{11}{ }^{31}$ Ideally, finite element analysis represents the best method to calculate circumferential stress, given the inhomogeneity of wall composition and mechanical properties in areas of vulnerable plaque. Intravascular ultrasound provides limited information on the distribution of the different components of 
atherosclerotic plaque, which has precluded use of the finite element technique in human subjects in vivo.

Peak systolic wall thickness measurements did not include the thickness of the arterial adventitia, which may lead to overestimation of circumferential stress. However, as the adventitia represents a small proportion of the thickness of an atherosclerotic coronary artery, overestimation of circumferential stress is likely to be minimal. In addition, it has been shown that the intima and media bear a greater proportion of total circumferential stress than the adventitia, ${ }^{11}$ implying intima-media thickness may be functionally more important when assessing wall stress in an artery with a circumferentially uniform structure.

In this current study we used a thin walled tube model to measure average circumferential stress in segments of artery with symmetrical thickening of the arterial wall rather than diseased segments with asymmetrical plaque. In segments of artery with asymmetrical plaque, mean circumferential wall stress is the same across the section containing plaque and the more normal artery wall, although the distribution of stress across different components of the vessel wall changes. In experimental studies, wall stress is greatest at the margins of the fibrous cap which overlies a lipid pool, ${ }^{11}$ and changes in this stress are most relevant to plaque rupture. Plaque cap stress cannot be measured directly with intravascular ultrasound, but in vitro studies have shown a good correlation between maximum stress determined by finite element analysis in asymmetrical vessels and plaque stress, calculated using a cylindrical tube model as the ratio of vessel radius and fibrous cap thickness. ${ }^{34}$ The mean reduction in coronary wall stress after esmolol of $\quad 0.17 \times 10^{6} \mathrm{dyn} / \mathrm{cm}^{2} \quad$ (approximately $125 \mathrm{~mm} \mathrm{Hg}$ ) was much smaller than the maximum circumferential stress of 4091 (1199) $\mathrm{mm} \mathrm{Hg}$ calculated at sites of plaque rupture in pathological specimens in vitro. ${ }^{31}$ However, it is possible that a small reduction in wall stress over prolonged periods decreases the risk of rupture when a plaque is vulnerable, because of weakening of the fibrous cap, increased lipid content, or local inflammation. Small differences in circumferential wall stress could therefore influence the risk of coronary events during longer term treatment.

It is important to understand the mechanisms by which treatment may influence the likelihood of coronary events. Intravenous esmolol is more effective than nicardipine at reducing circumferential coronary artery wall stress, a possible determinant of the risk of plaque rupture. This mechanism may in part explain the beneficial effects of $\beta$ blockers in clinical trials of patients with coronary artery disease. Results of large ongoing randomised clinical trials are awaited for reliable determination of the effects of treatment with dihydropyridine calcium antagonists on long term cardiovascular risk.

This study was supported in part by a University of Otago Research Grant and grants-in-aid from the Otago Medical
Research Foundation and The National Heart Foundation of New Zealand. Wyeth-Ayerst International Inc supplied the nicardipine. MJAW was supported, as the W and GS Dick research fellow, by the Southland Medical Foundation.

1 Yusuf S, Peto R, Lewis J, et al. Beta blockade during and after myocardial infarction: an overview of the randomized trials. Prog Card Dis 1985;27:335-71.

2 Dargie HJ, Ford I, Fox KM, for the TIBET study group. Total ischaemic burden European trial (TIBET): effects of ischaemia and treatment with atenolol, nifedipine SR and their combination on outcome in patients with chronic their combination on outcome in patients

3 Braun S, Boyko V, Behar S, et al. Calcium antagonists and mortality in patients with coronary artery disease: a cohort mortality in patients with coronary artery disease: a cohort

4 Furberg CD, Psaty BM, Meyer JV. Nifedipine. Dose-related increase in mortality in patients with coronary heart disease. Circulation 1995;92:1326-31.

5 Furberg CD, Psaty BM. Corrections to the nifedipine metaanalysis. Circulation 1996;93:1475-6.

6 Borhani NO, Mercuri M, Borhani PA, et al. Final outcome results of the multicenter isradipine diuretic atherosclerosis study (MIDAS): a randomized controlled trial. $7 A M A$ 1996;276:785-91.

7 Waters D. Proischemic complications of dihydropyridine calcium channel blockers. Circulation 1991;84:2598-600.

8 Packer M. Pathophysiological mechanisms underlying the adverse effects of calcium channel-blocking drugs in patients with chronic heart failure. Circulation 1989; 80(suppl IV):IV59-67.

9 Falk E. Why do plaques rupture? Circulation 1992;86(suppl III):III $30-42$

10 Falk E, Shah PK, Fuster V. Coronary plaque disruption. Circulation 1995;92:657-71.

11 Richardson PD, Davies MJ, Born GV. Influence of plaque configuration and stress distribution on fissuring of coronary atherosclerotic plaques. Lancet 1989;ii:941-4.

2 Potkin BN, Bartorelli AL, Gessert JM, et al. Coronary artery imaging with intravascular high-frequency ultrasound. Circulation 1990;81:1575-85.

13 Williams MJ, Stewart RA, Low CJ, et al. Assessment of the mechanical properties of coronary arteries using intravascular ultrasound: an in vivo study. Int f Card Imaging 1999; 15:287-94.

14 Carew TE, Vaishnav RN, Patel DJ. Compressibility of the arterial wall. Circ Res 1968;23:61-8.

15 Dobrin PB, Rovick AA. Influence of vascular smooth muscle on contractile mechanics and elasticity of arteries. Am $\mathcal{f}$ Physiol 1969;217:1644-51.

16 Langewouters GJ, Wesseling KH, Goedhard WJ. The static elastic properties of 45 human thoracic and 20 abdominal aortas in vitro and the parameters of a new model. 7 Biomech 1984;17:425-35.

17 Kaufmann P, Vassalli G, Utzinger U, et al. Coronary vasomotion during dynamic exercise: influence of intravenous and intracoronary nicardipine. $7 \mathrm{Am}$ Coll Cardiol 1995;26:624-31.

18 Bland JM, Altman DG. Statistical methods for assessing agreement between two methods of clinical measurement. Lancet 1986; ; $307-10$.

19 Estacio RO, Jeffers BW, Hiatt WR, et al. The effect of nisoldipine as compared with enalapril on cardiovascular outcomes in patients with non-insulin-dependent diabetes and hypertension. N Engl f Med 1998;338:645-52.

20 Psaty BM, Heckbert SR, Koepsell TD, et al. The risk of myocardial infarction associated with antihypertensive drug therapies. $\mathscr{F A M A}$ 1995;274:620-5.

21 Alderman $\mathrm{MH}$, Cohen $\mathrm{H}$, Roque $\mathrm{R}$, et al. Effect of long-acting and short-acting calcium antagonists on cardiovascular outcomes in hypertensive patients. Lancet 1997;349:594-8.

22 Grossman E, Messerli FH. Effect of calcium antagonists on plasma norepinephrine levels, heart rate, and blood pressure. Am f Cardiol 1997;80:1453-8.

23 Popov EP. Mechanics of materials, 2nd ed. Englewood Cliffs: Prentice-Hall, 1976.

24 Kjekshus JK. Importance of heart rate in determining betablocker efficacy in acute and long-term acute myocardial infarction intervention trials. Am 7 Cardiol 1986.57(suppl F): $43-9 F$.

25 Yusuf S. Verapamil following uncomplicated myocardial infarction: promising, but not proven. $\mathrm{Am} \mathcal{F}$ Cardiol 1996;77:421-2.

26 Hess OM, Bortone A, Gaglione A, et al. Effect of intracoronary and intravenous propranolol on human coronary nary and intravenous propranolol

27 Mittleman MA, Maclure M, Tofler GH, et al. Triggering of acute myocardial infarction by heavy physical exertion: protection against triggering by regular exertion. $N$ Engl $\mathcal{F}$ Med 1993;329:1677-83.

28 Willich SN, Lewis $M$, Lowel $\mathrm{H}$, et al. Physical exertion as a trigger of acute myocardial infarction. N Engl f Med 1993; 329:1684-90.

29 Arbab-Zadeh A, Penny WF, DeMaria AN, et al. Prevalence and extent of axial movement of intracoronary ultrasound transducer during the cardiac cycle [abstract]. Circulation 1996;94(suppl I):I-78

30 Loree HM, Kamm RD, Stringfellow RG, et al. Effects of fibrous cap thickness on peak circumferential stress in fibrous cap thickness on peak circumferential stress

31 Cheng GC, Loree HM, Kamm RD, et al. Distribution of circumferential stress in ruptured and stable atherosclerotic 
lesions: a structural analysis with histopathological correlation. Circulation 1993;87:1179-87.

32 Loree HM, Tobias BJ, Gibson LJ, et al. Mechanical properties of model atherosclerotic lesion lipid pools. Arterioscler Thromb 1994;14:230-4.
33 Libby P. Molecular bases of the acute coronary syndromes. Circulation 1995;91:2844-50.

34 Lee RT, Loree HM, Fishbein MC. High stress regions in saphenous vein bypass graft atherosclerotic lesions. $\mathcal{f} \mathrm{Am}$ Coll Cardiol 1994;24:1639-44.

\section{IMAGES IN CARDIOLOGY}

\section{Visualisation of ablated septal myocardium using myocardial contrast echocardiography in hypertrophic obstructive cardiomyopathy}

A 59 year old woman presented with exertional dyspnoea and presyncope. Cross sectional and Doppler echocardiography revealed asymmetric septal hypertrophy, systolic anterior movement of the anterior mitral leaflet, and left ventricular outflow obstruction with a pressure gradient of $140 \mathrm{~mm} \mathrm{Hg}$. She was diagnosed with hypertrophic obstructive cardiomyopathy (HOCM), and had been treated with $300 \mathrm{mg}$ of disopyramide per day. Nevertheless, her symptoms and pressure gradient had been refractory, so she was scheduled for percutaneous transluminal septal myocardial ablation (PTSMA). The first major septal branch of the left anterior descending artery was then probed with a 0.014 inch thick guide wire, and
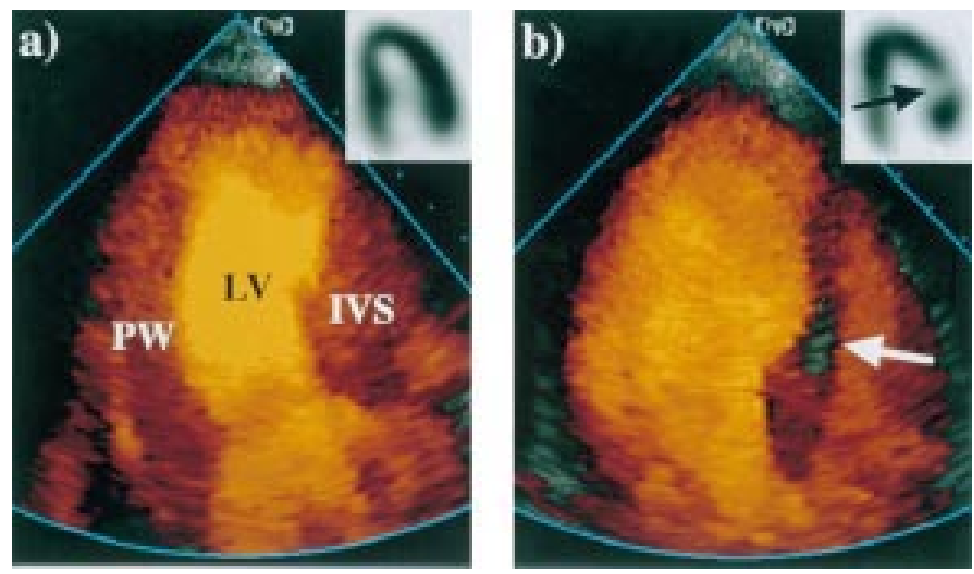

catheterised with a $1.5 \mathrm{~mm}$ angioplasty balloon catheter; $5 \mathrm{ml}$ of absolute alcohol was then slowly injected into the septal artery. After the procedure, the pressure gradient decreased from $140 \mathrm{~mm} \mathrm{Hg}$ to $50 \mathrm{~mm} \mathrm{Hg}$.

On the fifth postoperative day, echocardiographic studies were carried out again. A reduction in the pressure gradient remained, and new wall motion dyskinesia could be seen in the basal septum. We performed myocardial contrast echocardiography with ECG triggered end diastole harmonic power Doppler technique using intravenous Levovist (a saccharide based transpulmonary echo contrast agent; Schering AG) before and after PTSMA. The preoperative image demonstrated homogeneous myocardial opacification (left). In contrast, the postoperative image showed the contrast defect at the basal septum which was consistent with ${ }^{99 \mathrm{~m}}$ Tc- MIBI myocardial perfusion imaging (right).

PTSMA is an effective procedure for some HOCM patients who are symptomatic despite sufficient drug treatment. We suggest that myocardial contrast echocardiography using the ECG triggered harmonic power Doppler technique is useful for detecting ablated septal myocardium, as well as scintigraphy.

KEIJI HIROOKA MASAKAZU YAMAGISHI KUNIO MIYATAKE 